Copyright (C) 2006 IEEE. Reprinted from

IEEE Transactions on Communications, 2006; 54 (3):383-388

This material is posted here with permission of the IEEE. Such permission of the IEEE does not in any way imply IEEE endorsement of any of the University of Adelaide's products or services. Internal or personal use of this material is permitted. However, permission to reprint/republish this material for advertising or promotional purposes or for creating new collective works for resale or redistribution must be obtained from the IEEE by writing to pubs-permissions@ieee.org.

By choosing to view this document, you agree to all provisions of the copyright laws protecting it. 


\title{
Lower Bounds on the Minimal Delay of Complex Orthogonal Designs With Maximal Rates
}

\author{
Haibin Kan, Member, IEEE, and Hong Shen
}

\begin{abstract}
The maximal rates and the minimal delays are basic problems of space-time block codes from complex orthogonal designs. Liang systematically solved the problem on the maximal rates of complex othogonal designs, and posed an open problem on the minimal delays. Recently, the authors gave the negative answer for the open problem. In this letter, we give lower bounds on the minimal delays.
\end{abstract}

Index Terms-Complex orthogonal designs, delays, maximal rates, space-time block codes (STBCs).

\section{INTRODUCTION}

$\mathbf{S}$ PACE-TIME block codes (STBCs) from complex orthogonal designs have been hot topics in recent years, and are extensively applied in wireless communication systems with multiple transmit and receive antennas. The pioneer work in this field was done by Alamouti and Tarokh. Alamouti [1] first proposed a very simple and efficient transmission scheme using two transmit antennas and $m$ receive antennas, and his idea is the embryo of space-time codes (STCs) from orthogonal designs. Later, Tarokh [8] generalized Alamouti's idea to the general case, i.e., STCs from orthogonal designs. The STCs from orthogonal designs have full diversity and a linear maximum-likelihood (ML) decoding algorithm, which are important properties for wireless communication systems. The maximal rates and the minimal delays of orthogonal designs are two basic problems in this field. Tarokh proved that the maximal rates of orthogonal designs are not larger than one, gave a procedure for constructing real orthogonal designs with the maximal rate one and minimal delays, and also constructed a complex orthogonal design with rate $1 / 2$ from any real orthogonal design with rate 1 . Therefore, the problems on real orthogonal designs were completely solved. However, Tarokh pointed out in [8] that constructing complex orthogonal designs with a rate larger than $1 / 2$ was very difficult, and that his discoveries only presented the tip of the iceberg. Recently, Liang [4] made a great improvement on complex orthogonal designs. He systematically and

Paper approved by A. Anastasopoulos, the Editor for Wireless Communications of the IEEE Communications Society. Manuscript received August 13, 2004; revised May 24, 2005. This work was supported in part by the National Science Foundation of China under Grant 60472038, and in part by the Japan Society for Promotion of Science (JSPS) under Research Grant 17700008.

H. Kan is with the Department of Computer Science and Engineering, Fudan University, Shanghai, China. He is also with the Graduate School of Information Science, Japan Advanced Institute of Science and Technology, Ishikawa 9231292, Japan (e-mail: hbkan@fudan.edu.cn).

H. Shen is with the Graduate School of Information Science, Japan Advanced Institute of Science and Technology, Ishikawa 923-1292, Japan. He is also with the Department of Computer Science, University of Science and Technology of China, Hefei, China (e-mail: shen@jaist.ac.jp).

Digital Object Identifier 10.1109/TCOMM.2006.869880 smartly investigated the two basic problems on complex orthogonal designs, gave the maximal rates of complex orthogonal designs, and presented a procedure for constructing complex orthogonal designs with the maximal rates. Furthermore, he posed an open problem on the minimal delays of complex orthogonal designs with the maximal rates, and pointed out that the open problem was correct for not more than six transmit antennas. In [3], the authors proved that the open problem was also correct for seven transmit antennas, but gave as a counterexample the open problem for eight transmit antennas. In this letter, we give lower bounds for the minimal delays of orthogonal designs with the maximal rates.

Some preliminaries on orthogonal designs are given in Section II. We conclude the lower bounds on the minimal delays of complex orthogonal designs with the maximal rates in Section III, which are much smaller than the value in [4] and can be reached for $n=8$. Also, we point out that the lower bounds on the minimal delays are possibly reached for some even transmit antennas.

\section{PRELIMINARIES}

In this section, we introduce some basic notions on complex orthogonal designs. Let $C$ denote the complex field. All vectors are assumed to be column vectors. Denote by $C^{n}$ and $M_{m \times n}(C)$ the set of all $n$-dimensional complex vectors and the set of $m \times n$ complex matrices, respectively. For any $x=$ $\left(x_{1}, x_{2}, \ldots, x_{n}\right)^{t} \in C^{n}$, denote by $x^{t}=\left(x_{1}, x_{2}, \ldots, x_{n}\right)$, $x^{*}=\left(x_{1}^{*}, x_{2}^{*}, \ldots, x_{n}^{*}\right)^{t}$, and $x^{H}=\left(x_{1}^{*}, x_{2}^{*}, \ldots, x_{n}^{*}\right)$ its transpose vector, conjugate vector, and conjugate transpose vector, respectively. Similarly, for any matrix $A \in M_{m \times n}(C), A^{H}$ means the conjugate transpose of $A$. Denote by

$$
A\left(i_{1}, i_{2}, \ldots, i_{k} ; j_{1}, j_{2}, \ldots, j_{k}\right) \text { and } A\left(s_{1} \sim s_{2} ; t_{1} \sim t_{2}\right)
$$

the submatrix consisting of the $i_{1}$ th,$i_{2}$ th $, \ldots, i_{k}$ th rows and the $j_{1}$ th, $j_{2}$ th $, \ldots, i_{k}$ th columns of $A$, and the submatrix consisting of the $s_{1}$ th, $\left(s_{1}+1\right)$ th $, \ldots, s_{2}$ th rows and the $t_{1}$ th, $\left(t_{1}+1\right)$ th $, \ldots, t_{2}$ th columns of $A$, where $s_{1}<s_{2}$ and $t_{1}<t_{2}$, respectively. Sometimes, we denote by $A\left(i_{1}, i_{2}, \ldots, i_{k} ; t_{1} \sim t_{2}\right)$ the submatrix consisting of the $i_{1}$ th, $i_{2}$ th $, \ldots, i_{k}$ th rows and the $t_{1}$ th, $\left(t_{1}+1\right)$ th $, \ldots, t_{2}$ th columns of $A$. So $A(i ; j)$ denotes the $1 \times 1$ submatrix consisting of the $(i, j)$ element of $A$. We use $A(i, j)$ for the $(i, j)$ element of the matrix $A$. For any $x \in R,\lceil x\rceil$ and $\lfloor x\rfloor$ denote the least integer larger than or equal to $x$ and the largest integer less than or equal to $x$, respectively.

Definition 1: A $[p, n, k]$ complex orthogonal design $O$ is a $p \times n$ rectangular matrix whose nonzero entries are $z_{1}, z_{2}, \ldots, z_{k},-z_{1},-z_{2}, \ldots,-z_{k} \quad$ or 
$z_{1}^{*}, z_{2}^{*}, \ldots, z_{k}^{*},-z_{1}^{*},-z_{2}^{*}, \ldots,-z_{k}^{*}, \quad$ where $z_{1}, z_{2}, \ldots, z_{k}$, $z_{1}^{*}, z_{2}^{*}, \ldots, z_{k}^{*}$ are indeterminates over the complex number field $C$, such that

$$
O^{H} O=\left(\left|z_{1}\right|^{2}+\left|z_{2}\right|^{2}+\ldots+\left|z_{k}\right|^{2}\right) I_{n \times n} .
$$

When $p=n, O$ is called a square complex orthogonal design. $k / p$ is called the code rate of $O$, and $p$ is called the decoding delay of $O$.

According to Definition 1, it is easy to check that for a $[p, n, k]$ complex orthogonal design $O$, every column of $O$ contains exactly one of $z_{i},-z_{i}, z_{i}^{*}$, and $-z_{i}^{*}$ for each $i=1,2, \ldots, k$, and every row contains at most one of $z_{i},-z_{i}, z_{i}^{*}$, and $-z_{i}^{*}$ for each $i=1,2, \ldots, k$. If $O$ includes the following submatrix:

$$
\left(\begin{array}{ll}
z_{i} & s_{1} \\
s_{2} & z_{i}
\end{array}\right) \quad \text { or } \quad\left(\begin{array}{ll}
s_{1} & z_{i} \\
z_{i} & s_{2}
\end{array}\right)
$$

then $s_{1}=s_{2}=0$. If $O$ includes the following submatrix:

$$
\left(\begin{array}{ll}
z_{i} & s_{1} \\
s_{2} & z_{i}^{*}
\end{array}\right) \quad \text { or } \quad\left(\begin{array}{ll}
s_{1} & z_{i} \\
z_{i}^{*} & s_{2}
\end{array}\right)
$$

then $s_{2}=-s_{1}^{*}$.

Clearly, a $[p, n, k]$ complex orthogonal design $O$ is still a $[p, n, k]$ complex orthogonal design under the following transformations: 1) multiplication of rows or columns with -1 ; 2) permutation of rows or columns of $O$;3) permutation of complex variables in $O$; 4) multiplication of some complex variables with -1 ; 5) substitution of some complex variables in $O$ with their conjugates. Conventionally, we call them the elementary transformations for complex orthogonal designs.

In order to improve the bandwidth efficiency of the designed STBCs for any given number of transmit antennas, we need to construct complex orthogonal designs with rate $R$ as high as possible. Meanwhile, we should take into account the memory length or decoding delay $p$, which is expected to be as small as possible. So the following two problems are the basic problems in the study of complex orthogonal designs: 1) given $n$, find a $[p, n, k]$ complex orthogonal design which maximizes the rate $k / p ; 2)$ given $n$, find a $[p, n, k]$ complex orthogonal design with the maximal rate which minimizes the delay $p$. The first problem, i.e., the problem of maximal rates, was completely solved by Liang in [4]. Liang proved that for any given $n$, the maximal value of the rate $k / p$ of $[p, n, k]$ complex orthogonal designs is $m+1 / 2 m$, where $n=2 m$ or $2 m-1$. Also, he presented a procedure for constructing complex orthogonal designs with the maximal rates. In fact, for any given $n, m$, and $l$ such that $n=m+l$, a $[p(m, l), n, k(m, l)]$ complex orthogonal design can be constructed by Liang's procedure, where

$$
k(m, l)=\left(\begin{array}{l}
n \\
m
\end{array}\right) \text { and } p(m, l)=\left(\begin{array}{c}
n \\
m-1
\end{array}\right)+\left(\begin{array}{c}
n \\
m+1
\end{array}\right) .
$$

Furthermore, when $m=\lceil n / 2\rceil, k(m, l) / p(m, l)$ achieves the maximal value, i.e., $k(m, l) / p(m, l)=m+1 / 2 m$. So, when $n=2 m$, then

$$
k(m, m)=\left(\begin{array}{c}
2 m \\
m
\end{array}\right) \text { and } p(m, m)=\frac{2 m}{m+1}\left(\begin{array}{c}
2 m \\
m
\end{array}\right) .
$$

And when $n=2 m-1$, then

$$
\begin{aligned}
& k(m, m-1)=\left(\begin{array}{c}
2 m-1 \\
m
\end{array}\right) \\
& p(m, m-1)=\frac{2 m}{m+1}\left(\begin{array}{c}
2 m-1 \\
m
\end{array}\right) .
\end{aligned}
$$

However, the second basic problem, i.e., the problem of the minimal delays of complex orthogonal designs with maximal rates, is still an open problem. For any positive integer $n$ and real number $r$ not larger than one, denote by $\wp_{C}(n, r)$ the least positive integer $p$, such that there exists a $[p, n, k]$ complex orthogonal design with $k / p \geq r$. Given $n$, let $\gamma_{n}=m+1 / 2 m$, where $n=2 m$ or $2 m-1$. Liang [4] gave the following expression:

$$
\wp_{C}\left(n, \gamma_{n}\right)= \begin{cases}\frac{2 m}{m+1}\left(\begin{array}{c}
n \\
m
\end{array}\right), & \text { if } n=2 m \text { or } 2 m-1, \text { but } n \neq 4 \\
4, & \text { if } n=4\end{cases}
$$

and proved the above equation is correct for $1 \leq n \leq 6$. In [3], the authors proved the above equation is correct for $n=7$, but is not correct for $n=8$.

\section{LOWER BOUNDS ON THE MINIMAL DELAYS OF COMPLEX ORTHOGONAL DESIGNS WITH MAXIMAL RATES}

In this section, we deduce the lower bounds on the minimal delays of complex orthogonal designs with maximal rates and the lower bounds on the number of complex variables. Furthermore, we point out that the lower bounds on the minimal delays are possibly reached for some even transmit antennas.

Theorem 1: Given $n>4$, let $O$ be any $[p, n, k]$ complex orthogonal design with the maximal rate $m+1 / 2 m$, where $n=$ $2 m$ or $2 m-1$. Then

$$
p \geq n+\frac{1}{2} m(n-m)(n-2) .
$$

Proof: We only prove the case $n=2 m$, as it is completely similar to proving the case for $n=2 m-1$. Let $O$ be a $[p, n, k]$ complex orthogonal design with the maximal rate $m+1 / 2 m$. By the elementary transformations for complex orthogonal designs mentioned before, we could assume that $O$ contains the $n \times n$ submatrix $O_{0}$ in its top, where $O_{0}$ is given in (6), shown at the bottom of the next page. The condition $n>4$ and the proof of [4, Prop. 6] guarantee that the submatrix $O_{0}(1 \sim m ; m+1 \sim 2 m)$ should be occupied by pairwise different complex variables. Since $O$ is a complex orthogonal design, every column of $O$ should include every complex variable or its conjugate. In order to make every column include $z_{i}$, $-z_{i}, z_{i}^{*}$, or $-z_{i}^{*}$ for $2 \leq i \leq m+1, O$ must contain the following submatrix:

$$
\left(\begin{array}{l}
O_{0} \\
O_{1}
\end{array}\right)
$$

in its top by some elementary transformations, where $O_{1}$ is given in (7), and means an unoccupied position. It is easy to see that the number of rows of $O_{1}$ is

$$
\begin{aligned}
(m-1+m-1)+ & (m-1+m-2)+\ldots+ \\
(m-1+0) & =m(m-1)+\frac{1}{2} m(m-1) .
\end{aligned}
$$


Now, we prove that the unoccupied positions in $O_{1}$ can not be occupied by $\pm z_{i}$ and $\pm z_{i}^{*}, m+2 \leq i \leq m^{2}+1$. We first verify that the $(m-1) \times(m-1)$ submatrix $O_{1}(m \sim 2 m-2 ; 2 \sim m)$ does not contain $\pm z_{i}$ and $\pm z_{i}^{*}, m+2 \leq i \leq m^{2}+1$. Clearly

$$
O_{1}(m \sim 2 m-2 ; 2 \sim m)=O(3 m \sim 4 m-2 ; 2 \sim m)
$$

Since $O(m+1 \sim 2 m ; 2 \sim m)$ contains $-z_{i}^{*}, m+2 \leq i \leq$ $m^{2}+1, O_{1}(m \sim 2 m-2 ; 2 \sim m)$ does not contain $\pm z_{i}^{*}$, $m+2 \leq i \leq m^{2}+1$. Because

$$
O_{1}(m \sim 2 m-2 ; m+1)=\left(-z_{3}^{*},-z_{4}^{*}, \ldots,-z_{m+1}^{*}\right)^{t}
$$

and

$$
O_{0}(2 \sim m ; m+1)=\left(z_{m+2}, z_{2 m+2}, \ldots, z_{m(m-1)+2}\right)^{t}
$$

so $O_{1}(m \sim 2 m-2 ; 2 \sim m)$ does not contain $\pm z_{j m+2}$ for $1 \leq j \leq m-1$. Noting that $-z_{i}, m+2 \leq i \leq m^{2}+1$, have already appeared in the first column of $O_{1}$, we can use the following method to prove that $O_{1}(m \sim 2 m-2 ; 2 \sim m)$ does not include $\pm z_{h m+j}$, where $1 \leq h \leq m-1$ and $3 \leq$ $j \leq m+1$. For example, we verify that the third column of $O_{1}(m \sim 2 m-2 ; 2 \sim m)$ does not contain $\pm z_{m+3}$

$$
\begin{aligned}
& \text { Since } O(2,3)=0, O(2, m+2)=z_{m+3}, \\
& \text { and } O(3 m, m+2)=z_{2}^{*}, \quad O(3 m, 3) \neq \pm z_{m+3} .
\end{aligned}
$$

Assume $O_{1}(j, 3)=z_{m+3}$, where $m<j \leq 2 m-2$

$$
\begin{aligned}
& \text { Since } O_{1}(j, 3)=z_{m+3}, O_{1}(j, 2)=-z_{2 m+3}, \\
& O_{1}(2, j+2)=-z_{m+3}^{*}, \text { and } O_{1}(2, m+2)=z_{j+3}^{*} .
\end{aligned}
$$

Let $w=[(m-1+m-1)+\cdots+(m-1+m-(j-m+1))]+1$ Since $O_{1}(2, m+2)=z_{m+(j-m+3)}^{*}, O_{1}(w, 1)=-z_{j+3}$, and $O_{1}(2,1)=-z_{2 m+2}, O_{1}(w, m+2)=-z_{2 m+2}^{*}$.

Since $O_{1}(j, 2)=-z_{2 m+3}, O_{1}(w, 2)=z_{j-m+3}$, and $O_{1}(j, m+1)=-z_{j-m+3}^{*}, O_{1}(w, m+1)=-z_{2 m+3}^{*}$




where we get (9) by the submatrix $O_{0}$, (10) by the fact that $-z_{i}$, $m+2 \leq i \leq m^{2}+1$, have already appeared in the first column of $O_{1}$, and (11) by the fact that

$$
O_{1}(m \sim 2 m-2 ; m+1)=\left(-z_{3}^{*},-z_{4}^{*}, \ldots,-z_{m+1}^{*}\right)^{t} .
$$

So we have the following submatrix:

$$
O(3, w ; m+1, m+2)=\left(\begin{array}{cc}
z_{2 m+2} & z_{2 m+3} \\
-z_{2 m+3}^{*} & -z_{2 m+2}^{*}
\end{array}\right)
$$

which is a contradiction. Hence, $O_{1}(j, 3) \neq z_{m+3}$. Similarly, if $O_{1}(j, 3)=-z_{m+3}$, then we have the following submatrix:

$$
O(3, w ; m+1, m+2)=\left(\begin{array}{ll}
z_{2 m+2} & z_{2 m+3} \\
z_{2 m+3}^{*} & z_{2 m+2}^{*}
\end{array}\right)
$$

which is also a contradiction. Hence, $O_{1}(j, 3) \neq \pm z_{m+3}$. Similarly, we can prove that other $\pm z_{i}$ and $\pm z_{i}^{*}, m+4 \leq i \leq m^{2}+1$, do not appear in $O_{1}(m \sim 2 m-2 ; 2 \sim m)$. Consequently, $O_{1}(m \sim 2 m-2 ; 2 \sim m)$ does not include $\pm z_{i}$ and $\pm z_{i}^{*}$, $m+2 \leq i \leq m^{2}+1$. According to the procedure of (8)-(11), we can similarly verify that $\pm z_{i}$ and $\pm z_{i}^{*}, m+2 \leq i \leq m^{2}+1$, can not appear in the following submatrices:

$$
\begin{aligned}
& O_{1}((m-1+1) \sim(m-1+m-1) ; 2 \sim m) \\
& O_{1}([(m-1+m-1)+(m-1+1)] \\
& \quad \sim[(m-1+m-1)+(m-1+m-2)] ; 2 \sim m) \\
& \quad \ldots \\
& O_{1}([(m-1+m-1)+\cdots+(m-1+1)] ; 2 \sim m) .
\end{aligned}
$$

Furthermore, the above submatrices determine the other unoccupied positions in $O_{1}$. Hence, $O_{1}$ does not contain $\pm z_{i}$ and $\pm z_{i}^{*}, m+2 \leq i \leq m^{2}+1$. To make each column of $O$ contain $z_{i},-z_{i}, z_{i}^{*}$, or $-z_{i}^{*}, m+2 \leq i \leq 2 m+1$, we extend $O$ so that $O$ contains the following submatrix:

$$
\left(\begin{array}{l}
O_{0} \\
O_{1} \\
O_{2}
\end{array}\right)
$$

where $\mathrm{O}_{2}$ is defined in (12), shown at the bottom of the page. The number of rows of $\mathrm{O}_{2}$ is $m(m-2)+(1 / 2) m(m-1)$. Clearly, the unoccupied positions in the first column of $\mathrm{O}_{2}$ is completely determined by the elements in $O_{1}$. Noting that $-z_{i}$, $2 m+2 \leq i \leq m^{2}+1$, have already appeared in the second column of $\mathrm{O}_{2}$, we can similarly prove that the unoccupied positions in $\mathrm{O}_{2}$ do not include $z_{i},-z_{i}, z_{i}^{*}$, and $-z_{i}^{*}, 2 m+2 \leq i \leq$ $m^{2}+1$. Continuously, to make each column of $O$ contain $z_{i}$, $-z_{i}, z_{i}^{*}$, or $-z_{i}^{*}$ for $2 m+2 \leq i \leq m^{2}+1$, we conclude that $O$ has the following submatrix:

$$
\left(\begin{array}{c}
O_{0} \\
O_{1} \\
\vdots \\
O_{m}
\end{array}\right)
$$

where the number of rows of $O_{0}$ is $2 m$, and the number of the rows of $O_{i}, 1 \leq i \leq m$, is

$$
\begin{aligned}
(m-i+m-1)+ & (m-i+m-2)+\cdots+ \\
& (m-i+0)=(m-i) m+\frac{1}{2} m(m-1) .
\end{aligned}
$$

Hence, the number of rows of $O$ is not less than $2 m+\frac{1}{2} m^{2}(m-1)+\frac{1}{2} m^{2}(m-1)=n+\frac{1}{2} m(n-m)(n-2)$.

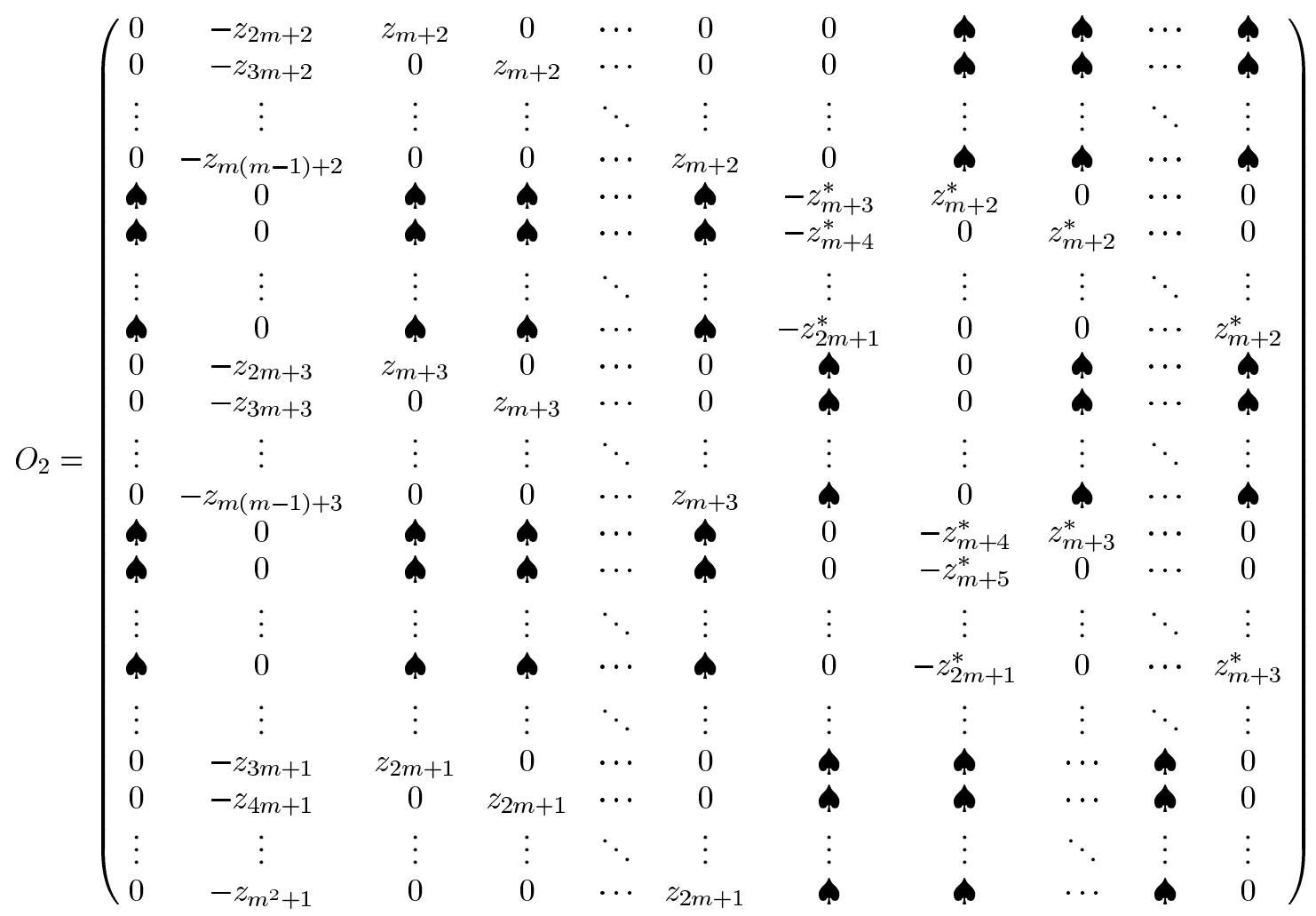


Thus, we finish the proof.

Clearly, for a given $n>4$, the lower bound in (5) is much less than the value given in (4). Kan and Shen [3] gave a [56, 8,35 ] complex orthogonal design, which shows that the lower bounds in (5) are reached when $n=8$.

Theorem 2: Given $n>4$, let $O$ be any $[p, n, k]$ complex orthogonal design with maximal rate $m+1 / 2 m$, where $n=2 m$ or $2 m-1$. Then

$$
k \geq 1+m(n-m)+\frac{1}{2}(m-1)(n-m)(n-m-1) .
$$

Proof: We only prove the case $n=2 m$, and the case $n=$ $2 m-1$ can be similarly proved. We remain the notations in the proof of Theorem 1. It has been proved that $\pm z_{i}$ and $\pm z_{i}^{*}$, $1 \leq i \leq m^{2}+1$, do not appear in $O_{1}$. Now we prove that all positions in the following submatrices of $O_{1}$ :

$$
\begin{aligned}
& O_{1}((m-1+1) \sim(m-1+m-1) ; 2 \sim m) \\
& O_{1}([m-1+m-1)+(m-1+1)] \\
& \quad \sim[(m-1+m-1)+(m-1+m-2)] ; 2 \sim m) \\
& \quad \ldots \\
& \left.O_{1}([m-1+m-1)+\cdots+(m-1+1)] ; 2 \sim m\right)
\end{aligned}
$$

should be occupied by new pairwise different complex variables. By Theorem 1 and the proof of [4, Prop. 6], we conclude that $O_{1}(m \sim 2 m-2 ; 2 \sim m)$ should be occupied by new pairwise different complex variables, say $z_{i}, m^{2}+2 \leq i \leq$ $2 m^{2}-2 m+2$. For simplicity of notation, let $z_{m^{2}+1+j}=y_{j}$ for $1 \leq j \leq(m-1)^{2}$, and let $s=m-1$. So we can assume that $O_{1}$ has the form given in (14), shown at the bottom of the page, where

$O_{1}(1 \sim m-1 ; m+2 \sim 2 m)=-\left(O_{1}(m \sim 2 m-2 ; 2 \sim m)\right)^{H}$.

Noting that the complex variables $y_{j}^{*}, 1 \leq j \leq s^{2}$, have already appeared in the $(m+1)$ th column of $O_{1}$, according to the procedure of (8)-(11), we can similarly prove that $y_{j},-y_{j}, y_{j}^{*},-y_{j}^{*}$, $1 \leq j \leq s^{2}$, do not appear in the following submatrices:

$$
\begin{aligned}
& O_{1}(3 m-2 \sim 4 m-5 ; 2 \sim m) \\
& O_{1}(5 m-5 \sim 6 m-9 ; 2 \sim m) \\
& \ldots \\
& O_{1}\left((m-1)^{2}+\frac{1}{2} m(m-1) ; 2 \sim m\right) .
\end{aligned}
$$

For example, we prove that $y_{j},-y_{j}, y_{j}^{*},-y_{j}^{*}, 1 \leq j \leq s^{2}$, do not appear in $O_{1}(3 m-2 \sim 4 m-5 ; 2 \sim m)$. Since every $y_{j}$, $1 \leq j \leq s^{2}$, appears in $O_{1}(m \sim 2 m-2 ; 2 \sim m)$, $\pm y_{j}$ for $1 \leq j \leq s^{2}$ can not appear in $O_{1}(3 m-2 \sim 4 m-5 ; 2 \sim m)$. Because

$$
O_{1}(3 m-2 \sim 4 m-5 ; m+2)=\left(-z_{4}^{*},-z_{5}^{*}, \ldots,-z_{m+1}^{*}\right)^{t}
$$

and

$$
O_{1}(1 \sim m-1 ; m+2)=\left(-y_{1}^{*},-y_{2}^{*}, \ldots,-y_{s}^{*}\right)^{t}
$$

so, $\pm y_{j}^{*}, 1 \leq j \leq s$, do not appear in $O_{1}(3 m-2 \sim 4 m-5 ; 2 \sim$ $m)$. Next, we prove that $\pm y_{j}^{*}, s+1 \leq j \leq s^{2}$, do not appear

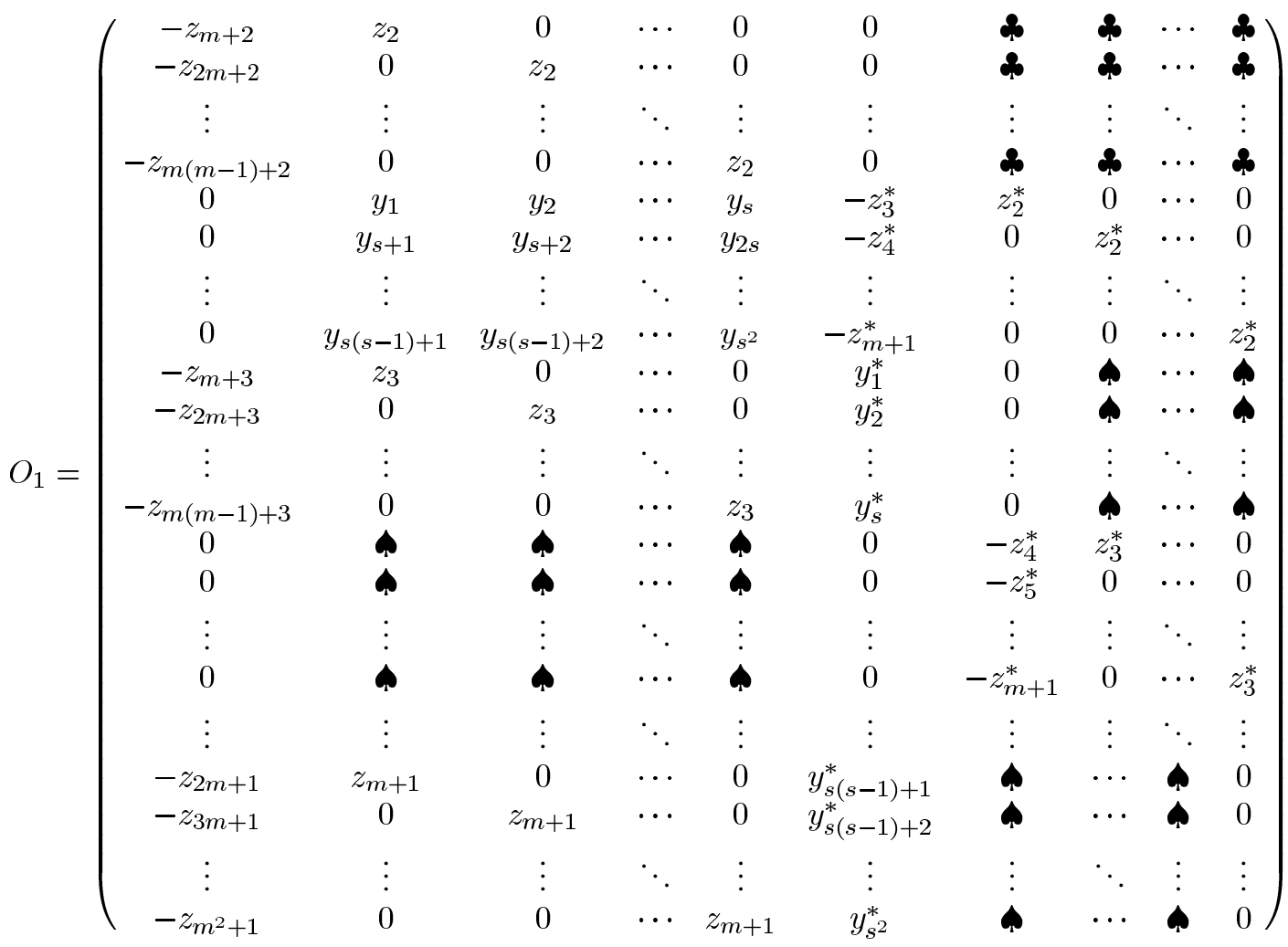


in $O_{1}(3 m-2 \sim 4 m-5 ; 2 \sim m)$. For instance, we verify that $\pm y_{s+1}^{*}$ do not appear in the second column of $O_{1}(3 m-2 \sim$ $4 m-5 ; 2 \sim m)$, as follows.

Since $O_{1}(1,3)=0, O_{1}(1, m+3)=-y_{s+1}^{*}$, and $O_{1}(3 m-$ $2, m+3)=z_{3}^{*}, O_{1}(3 m-2,3) \neq \pm y_{s+1}^{*}$. Assume $O_{1}(3 m-$ $1,3)=y_{s+1}^{*}$. Since $O_{1}(3 m-1,3)=y_{s+1}^{*}, O_{1}(3 m-1,2)=$ $-y_{s+2}^{*}, O_{1}(2 m, m+4)=-y_{s+1}$, and $O_{1}(2 m, m+3)=y_{2 s+1}$. Since $O_{1}(2 m, m+3)=y_{2 s+1}, O_{1}(6 m-8, m+1)=y_{2 s+1}^{*}$, and $O_{1}(2 m, m+1)=y_{2}^{*}, O_{1}(6 m-8, m+3)=-y_{2}$, where we make use of the important fact that $-y_{j}^{*}, 1 \leq j \leq s^{2}$, have already appeared in the $(m+1)$ th column of $O_{1}$. Since $O_{1}(3 m-1,2)=-y_{s+2}^{*}, O_{1}(3 m-1, m+2)=-z_{5}^{*}$, and $O_{1}(6 m-8,2)=z_{5}, O_{1}(6 m-8, m+2)=-y_{s+2}$. Hence, we have the following submatrix:

$$
O_{1}(2,6 m-8 ; m+1, m+2)=\left(\begin{array}{cc}
-y_{2}^{*} & -y_{s+2}^{*} \\
-y_{s+2} & -y_{2}
\end{array}\right)
$$

which is a contradiction. So $O_{1}(3 m-1,3) \neq y_{s+1}^{*}$. If $O_{1}(3 m-$ $1,3)=-y_{s+1}^{*}$, then we can get the following submatrix:

$$
O_{1}(2,6 m-8 ; m+1, m+2)=\left(\begin{array}{cc}
-y_{2}^{*} & -y_{s+2}^{*} \\
y_{s+2} & y_{2}
\end{array}\right)
$$

which is also a contradiction. Hence, $O_{1}(3 m-1,3) \neq \pm y_{s+1}^{*}$. In fact, the above procedure verifying that $\pm y_{s+1}^{*}$ do not appear in the second column of $O_{1}(3 m-2 \sim 4 m-5 ; 2 \sim m)$ is essentially the same as the procedure of (8)-(11). Since we can continuously do this procedure, we conclude that only new pairwise different complex variables appear in all positions of the following submatrix:

$$
\begin{aligned}
& O_{1}(m \sim 2 m-2 ; 2 \sim m) \\
& O_{1}(3 m-2 \sim 4 m-5 ; 2 \sim m) \\
& O_{1}(5 m-5 \sim 6 m-9 ; 2 \sim m) \\
& \cdots \\
& O_{1}\left((m-1)^{2}+\frac{1}{2} m(m-1) ; 2 \sim m\right) .
\end{aligned}
$$

Hence

$$
\begin{aligned}
k & \geq 1+m^{2}+(m-1)[(m-1)+(m-2)+\cdots+1] \\
& =1+m^{2}+\frac{1}{2}(m-1)^{2} m .
\end{aligned}
$$

Thus, we finish the proof.

The $[56,8,35]$ complex orthogonal design given in [3] shows that the lower bounds of Theorem 2 are reached for $n=8$. It is very easy to verify that when $n>4$, the lower bound for $p$ in Theorem 1 is larger than the lower bound for $k$ in Theorem 2 .

At last, we point out a very interesting property for the lower bounds in Theorems 1 and 2 . If $n=2 m$, then

$$
\begin{aligned}
1+ & m(n-m)+\frac{1}{2}(m-1)(n-m)(n-m-1) \\
& =1+m^{2}+\frac{1}{2} m(m-1)^{2} \\
& =\frac{1}{2}(m+1)^{2}(m-2)
\end{aligned}
$$

and

$$
\begin{aligned}
n & +\frac{1}{2} m(n-m)(n-2) \\
& =2 m+m^{2}(m-1) \\
& =m(m+1)(m-2) .
\end{aligned}
$$

Hence, if $n=2 m$, then

$$
\frac{1+m(n-m)+\frac{1}{2}(m-1)(n-m)(n-m-1)}{n+\frac{1}{2} m(n-m)(n-2)}=\frac{m+1}{2 m} .
$$

However, for given $n$, where $n=2 m, m+1 / 2 m$ is exactly the maximal rate of $[p, n, k]$ complex orthogonal designs. So, for an even integer $n$, where $n=2 m$ and $m \geq 4$, it is possible that the minimal delay of $[p, n, k]$ complex orthogonal designs is $m(m+1)(m-2)$, which is much less than the value given in (4).

\section{CONCLUSION}

Given a positive integer $n$, what the minimal delays are for complex orthogonal designs with maximal rates is an open problem. In this letter, for a positive integer $n$, we give lower bounds of the minimal delay $p$ and the number $k$ of complex variables for $[p, n, k]$ complex orthogonal designs with maximal rates. The lower bounds in Theorems 1 and 2 are weak, and it is not difficult to improve the lower bounds in Theorems 1 and 2 by a more careful analysis along with the same method in the letter. However, to completely solve the problem of the minimal delays for the complex orthogonal designs with maximal rates, the solution must appeal to other methods.

\section{ACKNOWLEDGMENT}

The authors would like to thank the referees for their careful reading and comments which have improved the clarity of this letter. They also thank Prof. Anastasopoulos for his kind help.

\section{REFERENCES}

[1] S. Alamouti, "A simple transmit diversity technique for wireless communications," IEEE J. Sel. Areas Commun., vol. 16, no. 7, pp. 1451-1458, Aug. 1998.

[2] R. Horn and C. Johnson, Matrix Analysis. New York: Cambridge Univ. Press, 1985.

[3] H.-B. Kan and H. Shen, "A counterexample for the open problem on the minimal delays of orthogonal designs with maximal rates," IEEE Trans. Inf. Theory, vol. 51, no. 1, pp. 355-359, Jan. 2005.

[4] X.-B. Liang, "Orthogonal designs with maximal rates," IEEE Trans. Inf. Theory, vol. 49, no. 10, pp. 2468-2503, Oct. 2003.

[5] X.-B. Liang and X.-G. Xia, "On the nonexistence of rate-one generalized complex orthogonal designs," IEEE Trans. Inf. Theory, vol. 49, no. 11, pp. 2984-2989, Nov. 2003.

[6] W. Su and X.-G. Xia, "Two generalized complex orthogonal space-time block codes of rates 7/11 and $3 / 5$ for 5 and 6 transmit antennas," IEEE Trans. Inf. Theory, vol. 49, no. 1, pp. 313-316, Jan. 2003.

[7] V. Tarokh, N. Seshadri, and N. Calderbank, "Space-time codes for high data rate wireless communication: Performance criterion and code construction," IEEE Trans. Inf. Theory, vol. 44, no. 2, pp. 744-765, Mar. 1998.

[8] V. Tarokh, H. Jafarkhani, and H. Calderbank, "Space-time codes from orthogonal designs," IEEE Trans. Inf. Theory, vol. 45, no. 5, pp. 1456-1467, Jul. 1999.

[9] — "Space-time block coding for wireless communications: Performance results," IEEE J. Sel. Areas Commun., vol. 17, no. 3, pp. 451-460, Mar. 1999.

[10] H. Wang and X.-G. Xia, "Upper bounds of rates of complex orthogonal space-time block codes," IEEE Trans. Inf. Theory, vol. 49, no. 10, pp. 2788-2796, Oct. 2003. 